OPEN ACCESS

Edited by:

David W. Ussery,

University of Arkansas for Medical

Sciences, United States

Reviewed by:

Delio José Mora Junior, Federal University of Southern Bahia,

Brazil

Patricia Escandon

Instituto Nacional de Salud, Colombia

*Correspondence:

Abdalla Ahmed

aoahmed@gmail.com

${ }^{t}$ These authors have contributed equally to this work

Specialty section:

This article was submitted to

Evolutionary and Genomic

Microbiology,

a section of the journal

Frontiers in Microbiology

Received: 16 June 2021 Accepted: 31 August 2021

Published: 11 October 2021

Citation:

Sirag B, Khidir E-S, Dumyati M, Sindi B, Alsinnari M, Faidah $\mathrm{H}$ and

Ahmed A (2021) Cryptococcus neoformans and Other Opportunistic

Cryptococcus Species in Pigeon

Dropping in Saudi Arabia: Identification and Characterization by

DNA Sequencing.

Front. Microbiol. 12:726203.

doi: 10.3389/fmicb.2021.726203

\section{Cryptococcus neoformans and Other Opportunistic Cryptococcus Species in Pigeon Dropping in Saudi Arabia: Identification and Characterization by DNA Sequencing}

Bashir Sirag ${ }^{1+}$, El-Shiekh Khidir ${ }^{2+}$, Mohammed Dumyati ${ }^{3}$, Basam Sindi ${ }^{4}$, Mahir Alsinnari ${ }^{5}$, Hani Faidah ${ }^{1}$ and Abdalla Ahmed ${ }^{1 *}$

\begin{abstract}
' Department of Microbiology, College of Medicine, Umm Al-Qura University, Makkah, Saudi Arabia, ${ }^{2}$ Laboratory Medicine Department, Faculty of Applied Medical Sciences, Umm Al-Qura University, Makkah, Saudi Arabia, ${ }^{3}$ Department

of Medicine, National Guard Health Affairs, King Abdulaziz Medical City, Jeddah, Saudi Arabia, ${ }^{4}$ Department of Medicine, King Fahad Armed Forces Hospital, Jeddah, Saudi Arabia, ${ }^{5}$ Department of Anesthesia, Al Noor Specialist Hospital, Makkah, Saudi Arabia
\end{abstract}

The prevalent variants of Cryptococcus neoformans, and other Cryptococcus species in pigeon excreta in Western Region of Saudi Arabia were studied. Ninety pigeon dropping samples were plated directly on Niger seed agar, and suspected colonies were sequenced using Illumina MiSeq. Species identification was determined using sequence read mapping to reference genomes of the two $C$. neoformans variants. In addition, sequence reads were identified using the KmerFinder tool. internal transcribed spacer 2 in the rDNA was also used for fungal barcoding of none of the C. neoformans species using two fungal identification databases. Phylogeny was studied using CSI Phylogeny (Center for Genomic Epidemiology, Denmark). The C. neoformans var. grubii mitochondrion and chromosome 1 reference sequences (accession numbers NC_004336.1 and CP022321.1, respectively) were used for sequence comparison and variant calling. Fifteen Cryptococcus isolates were isolated, 11 were identified as C. neoformans var. grubii, and 4 were found to be other opportunistic Cryptococcus species. Phylogeny analysis of $C$. neoformans var. grubii isolates showed a high degree of similarity between the $C$. neoformans isolates especially at the mitochondrial genome level. This study supports the fact that pathogenic and opportunistic Cryptococcus species are prevalent in domestic bird excreta which is an easy source of infection in the susceptible population.

Keywords: Cryptococcus neoformans, pigeon dropping, Saudi Arabia, DNA sequencing, KmerFinder

\section{INTRODUCTION}

Cryptococcus neoformans and Cryptococcus gattii are exogenous fungal pathogens with C. neoformans commonly associated with infections in immunocompromised patients, while C. gattii affects predominantly immunocompetent individuals (Girish Kumar et al., 2010). The sexual form of C. neoformans, Filobasidiella neoformans, is a filamentous fungus often found in 
pigeon excrement, while the sexual form of C. gattii, Filobasidiella bacillispora, lives mainly in certain types of trees. Most infections with $C$. neoformans consist of a lung infection. However, fungal meningitis and encephalitis, especially as a secondary infection for AIDS patients, are often caused by C. neoformans, making it a particularly dangerous fungus. There are several research groups focusing on the molecular determination of the number of genetically diverse subgroups within each Cryptococcus species. The molecular methods employed by each group to construct phylogenetic trees vary, and different methods have resulted in different numbers of subgroups. Interestingly, an association between geographic origin and certain genotypes has been observed, implying epidemiological significance of certain genotypes (Satoh et al., 2011; Chae et al., 2012a,b; Qishui et al., 2012; Wu et al., 2012).

Cryptococcus neoformans, the agent of cryptococcosis, had been considered a homogeneous species until 1949 when the existence of four serotypes was revealed based on the antigenic properties of its polysaccharide capsule. Such heterogeneity of the species, however, remained obscure until the two morphologically distinct teleomorphs of C. neoformans were discovered during the mid-1970s. The teleomorph F. neoformans was found to be produced by strains of serotypes A and D while F. bacillispora was found to be produced by strains of serotypes B and C. Latter studies revealed numerous differences between the anamorphs of the two Filobasidiella species with regard to their ecology, epidemiology, pathobiology, biochemistry, and genetics. Presently, the etiologic agent of cryptococcosis is classified into two species, C. neoformans (serotypes A and D) and C. gattii (serotypes $\mathrm{B}$ and $\mathrm{C}$ ). Intraspecific genetic diversity has also been revealed as more genotyping methods have been applied for each serotype (Sugita et al., 2000). As a result, the number of scientifically valid species within C. neoformans has become a controversial issue because of the differing opinions among taxonomists as to the appropriate definition of a species.

Due to the availability and affordability of better-resolution molecular biology techniques, such as DNA sequencing, more reports of infections with previously less reported Cryptococcus species become more evident (Yong et al., 2016; Pakshir et al., 2019; Mirpourian et al., 2021). Other less common opportunistic Cryptococcus species are becoming an important cause of serious infections such as peritonitis and fungaemia in immunocompromised patients (Ragupathi and Reyna, 2015; Aghaei Gharehbolagh et al., 2017; Choe et al., 2020; RodriguezLeguizamon et al., 2020). These infections might not be an emerging infection; however, the availability of whole-genome sequence data and the availability of easy-to-use bioinformatics tools are helping medical microbiology laboratories to better detect and identify these opportunistic Cryptococcus species (Vajpeyi and Chandran, 2016).

The aim of this study is to identity the prevalent cryptococcus variants of $C$. neoformans in pigeon dropping in Makkah Region in Saudi Arabia using DNA sequencing. No wholegenome sequence data are currently available from isolates from this region, and therefore, nothing is known about circulating species and genotypes. Knowing the prevalent genotypes will help in the understanding of Cryptococcus virulence, pathogenesis, and susceptible population. In this study, the natural ecological niches of $C$. neoformans were explored and isolated species was studied by DNA sequencing. Molecular data of C. neoformans from our region will help in defining the molecular epidemiology and taxonomy of this important opportunistic fungal species.

\section{MATERIALS AND METHODS}

\section{Specimen Collection and Yeast Isolation}

Specimens were collected from pigeon nets and pigeons' feeding areas, where fresh and dry pigeons' droppings are available in big quantities. Ninety pigeon dropping specimens were collected from the Makkah region including specimens from Makkah Holy City, Taif, and Jeddah. Specimens were collected in 30-ml sterile plastic containers and stored at room temperature. One gram of each specimen was suspended in sterile physiological saline supplemented with chloramphenicol at a final concentration of $0.1 \%(\mathrm{w} / \mathrm{v})$. Serial 10 -fold dilutions, in the same physiological solution, were prepared and used for direct isolation of Cryptococcus species. Culture was done on selective Sabouraud Dextrose Agar and Niger Seed agar and incubated for 3-5 days at room temperature. Capsulated pigmented (melanin producing) yeast colonies on Niger seed agar were presumptively identified as Cryptococcus. These types of yeast colonies were purified by subculture in Sabouraud Dextrose Agar, and India Ink staining was used to screen for capsule production. Yeasts colonies were purified and stored at $20 \%$ glycerol medium at $-20^{\circ} \mathrm{C}$.

\section{DNA Extraction and Genome Sequencing}

Yeast cells from fresh cultures were used for DNA extraction. Cells were harvested from 2- to 3-day-old cultures and washed using sterile Tris-EDTA buffer (TE) pH 8.0 in 2-ml screw cap tubes and then resuspended in $500 \mu 1 \mathrm{TE}$ buffer. The cell wall was disrupted using 0.5-mm glass beads in BioSpec Mini-Beadbeater16 (BioSpec Inc., Bartlesville, OK, United States) for $5 \mathrm{~min}$ then cooled in ice for an additional $5 \mathrm{~min}$. The aqueous layer containing DNA was separated from proteins and cell debris using two phenol/chloroform (1:24 pH 8.0) extractions. DNA was then precipitated by isopropanol, washed with $70 \%$ ethanol, dried at room temperature, and resuspended into $35 \mu \mathrm{TE}$ buffer $\mathrm{pH}$ 8.0. The quantity and quality of the isolated DNA were determined using Qubit ${ }^{\circledR}$ (Invitrogen, Applied Biosystems, Carlsbad, CA, United States) and Agilent 2100 Bioanalyzer using DNA 1000 Chip (Agilent Inc., Santa Clara, CA, United States).

\section{Library Preparation for DNA Sequencing}

Genomic DNA libraries were prepared using Illumina Nextera XT Library Preparation Kit, and samples were barcoded using Nextera XT Index Kit (Illumina Inc., San Diego, CA, United States). DNA sequencing libraries were prepared using $1 \mathrm{ng}$ input genomic DNA and validated and quantified directly without normalization using Agilent Bioanalyzer 2100 High Sensitivity DNA Chip (Agilent Inc., United States). Cryptococcus genomes were sequenced in Illumina MiSeq using the paired-end 


\section{Cryptococcus neoformans var. grubii}

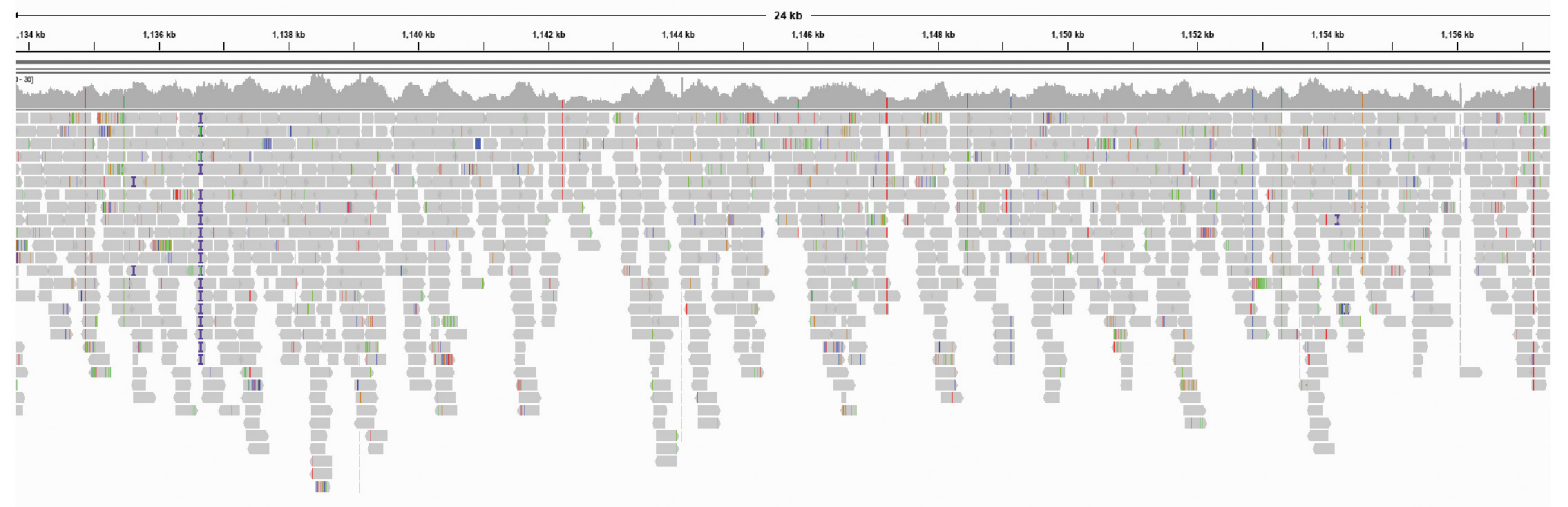

\section{Cryptococcus neoformans var. neoformans}

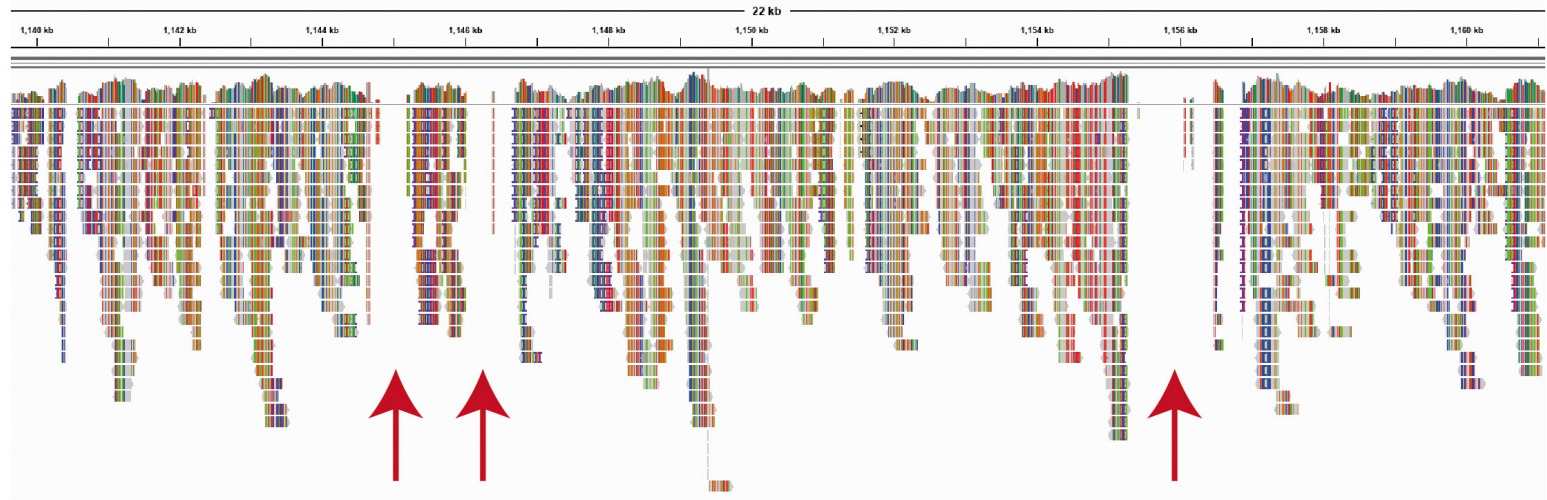

FIGURE 1 | Mapping of isolate C2 sequence reads as visualized by the Integrative Genomics Viewer (IGV) version 2.8 .2 (Broad Institute, Cambridge, MA, United States). Sequence reads of isolate C2 were mapped using BWA-MEM, which maps medium and long reads (>100 bp). Reference-guided mapping was performed using chromosome 1 of each Cryptococcus neoformans variety (accession numbers NC_006670.1 and CP022321.1, respectively) as a reference genome. Mapping was performed using BWA-MEM (Galaxy Version 0.7.17.1), and BAM files were visualized by the IGV, which show even template coverage in the case of Cryptococcus neoformans var. grubii, while in the case of Cryptococcus neoformans var. neoformans you see clear gabs in the template coverage indicated by red arrows.

protocol and version-3 600-cycle kit. The quality of the pairedend sequence reads was checked by FastQC (Galaxy Version $0.72+$ galaxy1) before subsequent data analysis.

\section{Molecular Identification}

Molecular identification was done by mapping of the sequence reads to the two possible reference genomes of $C$. neoformans variants (neoformans and grubii). Mapping was done using BWA-MEM, which maps medium and long reads (>100 bp). Reference-guided mapping was performed using chromosome 1 of each C. neoformans variety (accession numbers NC_006670.1 and CP022321.1, respectively) as a reference genome. Mapping was performed using BWA-MEM (Galaxy Version 0.7.17.1), and BAM files were visualized by the Integrative Genomics Viewer (IGV) version 2.8.2 (Broad Institute, Cambridge, MA, United States).

Species identification was also confirmed using KmerFinder version 3.0.2 from the Center for Genomic Epidemiology. KmerFinder predicts microbial species using a fast $K$-mer algorithm (Hasman et al., 2014; Larsen et al., 2014; Clausen et al., 2018). FastQ files of all the isolated yeast were used for the $K$-mer identification.

Isolates which showed poor mapping to $C$. neoformans reference genomes or showed poor $K$-mer scores with KmerFinder were identified based on their rDNA sequences. The internal transcribed spacer (ITS) sequences were obtained by direct Illumina sequencing, and species were identified using the UNITE ITS Database ${ }^{1}$ and the ISHAM ITS Database ${ }^{2}$. To get the most reliable identification, only the ITS 2 sequences were used for species identification.

\section{Phylogenetic Analysis}

Phylogeny was studied using CSI Phylogeny (Center for Genomic Epidemiology, Denmark). CSI Phylogeny calls SNPs, filters the SNPs, does site validation, and infers a phylogeny based on the concatenated alignment of the high-quality SNPs

\footnotetext{
${ }^{1}$ https://unite.ut.ee/

${ }^{2}$ https://its.mycologylab.org/
} 
(Delcher et al., 2002; Li and Durbin, 2009; Li et al., 2009; Price et al., 2010; Quinlan and Hall, 2010; Kaas et al., 2014). The C. neoformans var. grubii mitochondrion and chromosome 1 reference sequences (accession number NC_004336.1 and CP022321.1, respectively) were used for sequence comparison and variant calling.

\section{RESULTS}

Fifteen Cryptococcus isolates were isolated, and the yeast species identity was confirmed using DNA whole-genome sequencing with low sequence coverage $(\times 20$ to $\times 30)$ enough to map the sequence reads to the possible reference genomes. The sequence mapping showed that the closer genome for the majority of the isolates $(\mathrm{C} 1-\mathrm{C} 11,73 \%)$ was $C$. neoformans var. grubii (Figure 1). Mapping showed even template coverage in case of $C$. neoformans var. grubii, while in case of $C$. neoformans var. neoformans the reference genome coverage was not complete with many gabs, as shown in Figure 1.

The species identification of C. neoformans var. grubii was also confirmed by the KmerFinder tool. High $K$-mer matching scores were found with all C. neoformans var. grubii chromosomes. The chromosome coverage ranged from 86 to $93 \%$, and the sequencing depths ranged from $\times 15$ to $\times 21$ (Table 1). The $K$-mer matching score showed $100 \%$ template coverage and very high sequencing depth in case of the mitochondrial genome of C. neoformans var. grubii (Table 1).

Based on C. neoformans var. grubii chromosome 1 (accession number CP022321.1), phylogeny analysis showed high degree of similarity of most of the isolates, in which 10 out of 11 were clustered in the same branch together with the reference chromosome sequence. However, in case of phylogeny based on the C. neoformans var. grubii mitochondrion genome (accession number NC_004336.1), different isolates were found to be clustering in different branches in the phylogenetic tree (Figure 2).

Four out of the 15 isolates were not identified as C. neoformans. Sequencing of the ITS 2 identified these isolates as Naganishia albidus (formerly, Cryptococcus albidus) Naganishia diffluens, Naganishia liquefaciens, Naganishia albidosimilis, and Naganishia globose, which are known opportunistic Cryptococcus species (Table 2).

\section{DISCUSSION}

Many studies have recently used the whole-genome sequence data of Cryptococcus species to better understand the evaluation, genotype relations, diversity, pathogenicity, and antifungal susceptibility of this important genus and to explore other none Cryptococcus yeasts associated with pigeon dropping (Heitman et al., 1999; Florio et al., 2011; Gillece et al., 2011; Engelthaler et al., 2014; Meyer, 2015; Movahed et al., 2015; Lockhart et al., 2016). Some of these studies were carried out on C. neoformans and its sister species Cryptococcus gattii, which is an emerging pathogen connected with the ongoing cryptococcosis outbreak on Vancouver Island (Meyer, 2015; Lockhart et al., 2016).

In this study, we screened 80 environmental samples representing different areas in the Western Region in Saudi Arabia. We managed to sequence the whole genome of all positive cultures (number 15 isolates) as the only data of its type from this region. The sequence data allowed the identification of the less common opportunistic Cryptococcus species, confirming the variety of the pathogenic C. neoformans as $C$. neoformans vari. grubii.

As expected, C. neoformans was easily isolated by direct culture of pigeon dropping (Chae et al., 2012a,b; Soltani et al.,

TABLE 1 | Results of identification of Cryptococcus isolate number C2 using KmerFinder bioinformatic tool.

\begin{tabular}{|c|c|c|c|c|}
\hline \# Assembly & $K$-mer matching score & Template coverage $\%$ & Depth & Reference sequences \\
\hline GCF_000149245.1 & $1,402,196$ & 94 & 17 & Cryptococcus neoformans var. grubii H99 chromosome 1 \\
\hline GCF_000149245.1 & $1,249,263$ & 93 & 21 & Cryptococcus neoformans var. grubii H99 chromosome 2 \\
\hline GCF_000149245.1 & 922,974 & 93 & 16 & Cryptococcus neoformans var. grubii H99 chromosome 3 \\
\hline GCF_000149245.1 & 650,005 & 90 & 16 & Cryptococcus neoformans var. grubii H99 chromosome 4 \\
\hline GCF_000149245.1 & $1,033,422$ & 92 & 15 & Cryptococcus neoformans var. grubii H99 chromosome 5 \\
\hline GCF_000149245.1 & 854,952 & 93 & 16 & Cryptococcus neoformans var. grubii H99 chromosome 6 \\
\hline GCF_000149245.1 & 820,242 & 91 & 16 & Cryptococcus neoformans var. grubii H99 chromosome 7 \\
\hline GCF_000149245.1 & 798,403 & 91 & 15 & Cryptococcus neoformans var. grubii H99 chromosome 8 \\
\hline GCF_000149245.1 & 707,589 & 91 & 16 & Cryptococcus neoformans var. grubii H99 chromosome 9 \\
\hline GCF_000149245.1 & 637,173 & 90 & 16 & Cryptococcus neoformans var. grubii H99 chromosome 10 \\
\hline GCF_000149245.1 & 903,131 & 91 & 16 & Cryptococcus neoformans var. grubii H99 chromosome 11 \\
\hline GCF_000149245.1 & 458,861 & 88 & 16 & Cryptococcus neoformans var. grubii H99 chromosome 12 \\
\hline GCF_000149245.1 & 464,331 & 86 & 16 & Cryptococcus neoformans var. grubii H99 chromosome 13 \\
\hline GCF_000149245.1 & 581,666 & 89 & 17 & Cryptococcus neoformans var. grubii H99 chromosome 14 \\
\hline GCF_000149245.1 & $1,707,609$ & 100 & 1809 & Cryptococcus neoformans var. grubii H99 mitochondrion \\
\hline
\end{tabular}

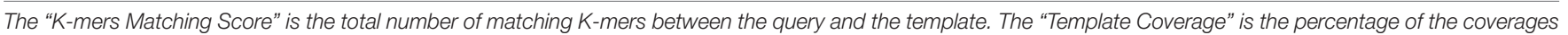

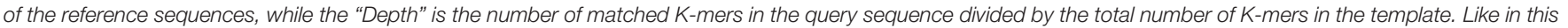
case, when using read files "Depth," this estimates the sequencing depth. 


\section{Cryptococcus neoformans var. grubii}
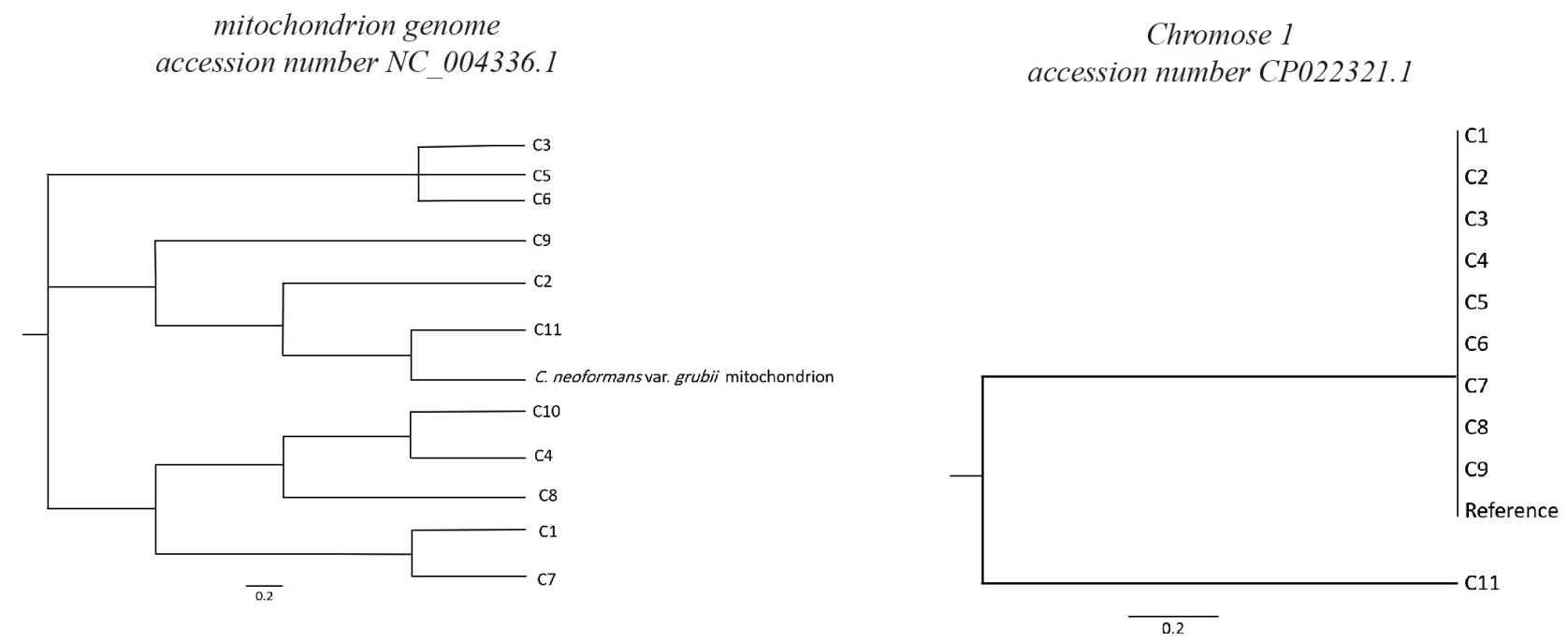

FIGURE 2 | Phylogeny of Cryptococcus neoformans var. grubii isolates. Sequence read analysis was done using CSI Phylogeny (Center for Genomic Epidemiology, Denmark). CSI Phylogeny calls SNPs, filters the SNPs, does site validation, and infers a phylogeny based on the concatenated alignment of the high-quality SNPs. The Cryptococcus neoformans var. grubii mitochondrion and chromosome 1 reference sequences (accession number NC_004336.1 and CP022321.1, respectively) were used for sequence comparison and variant calling. Phylogeny analysis showed high degree of similarity of most of the isolates (10 out of 11) to Cryptococcus neoformans var. grubii chromosome 1, while analysis based on sequences comparison to the mitochondrion genome showed varied degrees of similarities.

TABLE 2 | Species identification using the UNITE ITS database and ISHAM ITS database.

\begin{tabular}{lllll}
\hline Isolate \# & Unite database & ID \% & ISHAM database & ID\% \\
\hline C12 & Naganishia albida & 96.8 & Cryptococcus albidus & 93.6 \\
& Naganishia diffluens & 96.8 & & \\
& Naganishia liquefaciens & 96.8 & & \\
C13 & Naganishia adeliensis & 96.8 & & 90.4 \\
& Naganishia albida & 95.8 & Cryptococcus albidus & \\
& Naganishia diffluens & 95.8 & & \\
& Naganishia liquefaciens & 95.8 & & \\
C14 & Naganishia albidosimilis & 95.8 & & 96.8 \\
& Naganishia albida & 98.4 & Cryptococcus albidus & \\
& Naganishia diffluens & 98.4 & Naganishia diffluens & 96.8 \\
& Naganishia liquefaciens & 98.4 & & \\
& Naganishia albidosimilis & 98.4 & & \\
C15 & Naganishia albida & 99.2 & Cryptococcus albidus & 96.0 \\
& Naganishia diffluens & 99.2 & Naganishia diffluens & 96.0 \\
& Naganishia liquefaciens & 99.2 & & \\
& Naganishia albidosimilis & 99.2 & & \\
& Naganishia globosa & 99.2 & &
\end{tabular}

To get the most reliable identification, only the ITS 2 sequences were used for species identification. Based on ITS 2 sequences, the closely related species were not differentiated even at the secondary structure levels. ${ }^{*}$ Cryptococcus albidus is a synonym of Naganishia albida.

2013; Ellabib et al., 2016; Ghaderi et al., 2019; Pakshir et al., 2019; Mirpourian et al., 2021) and identification was confirmed by DNA sequencing. Sequence-based identification was confirmed by different ways. Reference genome-guided mapping of raw sequences is one way of the well-known approaches for species identifications of unknown sequence reads. Most of the isolates showed good even mapping without gabs when using the C. neoformans var. grubii H99 chromosome 1 reference sequence, while less template coverage was observed when using C. neoformans var. neoformans H99 chromosome 1 reference sequence (Figure 1). In addition, species was also confirmed using the KmerFinder which gave high scores of $K$-mer matching with chromosomal and mitochondrial genomes of $C$. neoformans var. grubii.

The analysis of the whole-genome sequence data of none of the C. neoformans isolates $(n=4)$ showed low scores of matching $K$-mers. These four isolates that showed no matching of $K$-mers with the fungal database were identified based on ITS2 similarity as N. albidus (formerly, C. albidus) N. diffluens, N. liquefaciens, $N$. albidosimilis, and $N$. globose. The identification of these less common opportunistic Cryptococcus species is becoming much easier with the availability and affordability of PCR and/or ITS sequencing for most of microbiology laboratories (Chae et al., 2012a,b; Ellabib et al., 2016; Dou et al., 2017).

We were not able to identify the less common Cryptococcus species using the KmerFinder tool due to lack of genome sequence data in the KmerFinder database. N. albidus have a draft genome record in GenBank, which has not yet been updated in the KmerFinder database (Vajpeyi and Chandran, 2016; Yong et al., 2016). Many reports showed the clinical importance of these species as opportunistic pathogens (Ragupathi and Reyna, 2015; Aghaei Gharehbolagh et al., 2017; 
Choe et al., 2020; Rodriguez-Leguizamon et al., 2020); however, none of these less common C. neoformans has been reported from this region before.

Due to similarities of morphology and ecological niches of these environmental basidiomycetous yeasts, it is always recommended to use the DNA-based method for species identification. DNA-based identification and typing are becoming the standard method of Cryptococcus species identification in many laboratories (Satoh et al., 2011; Chae et al., 2012b); however, scientists should always be careful and should always use tools that allow identification of all possible pathogens.

Although DNA-based methods are becoming popular and accessible to many laboratories, less common Cryptococcus opportunistic species can be easily missed even if DNA sequencing is used for species identification. This is because of lack of reference genomes sequence data which is needed for identification by bioinformatics tools such as KmerFinder. The identification will be more confusing when only a few sets of markers, such as ITS region, are used.

In this study, the less common Cryptococcus opportunistic species were poorly identified when only ITS2 sequences were used. In four isolates, more than one species was predicted and it was not possible to differentiate between Naganishia species (Table 2). Identifications could be improved by adding more markers, but the best way to achieve proper identification is by updating the identification databases of tools such as KmerFinder with whole-genome sequence data of these less common opportunistic yeasts species.

We conclude that $C$. neoformans var. grubii is the most common variety of $C$. neoformans in pigeon drooping in

\section{REFERENCES}

Aghaei Gharehbolagh, S., Nasimi, M., Agha Kuchak Afshari, S., Ghasemi, Z., and Rezaie, S. (2017). First case of superficial infection due to Naganishia albida (formerly Cryptococcus albidus) in iran: a review of the literature. Curr. Med. Mycol. 3, 33-37. doi: 10.18869/acadpub.cmm.3.2.33

Chae, H. S., Jang, G. E., Kim, N. H., Son, H. R., Lee, J. H., Kim, S. H., et al. (2012a). Classification of Cryptococcus neoformans and yeast-like fungus isolates from pigeon droppings by colony phenotyping and ITS genotyping and their seasonal variations in Korea. Avian Dis. 56, 58-64.

Chae, H. S., Park, G. N., Kim, S. H., Jo, H. J., Kim, J. T., Jeoung, H. Y., et al. (2012b). Rapid direct identification of Cryptococcus neoformans from pigeon droppings by nested PCR using CNLAC1 gene. Poult. Sci. 91, 1983-1989. doi: 10.3382/ps.2012-02307

Choe, Y. J., Blatt, D. B., Yalcindag, A., Geffert, S. F., Bobenchik, A. M., and Michelow, I. C. (2020). Cryptococcus albidus fungemia in an immunosuppressed child: case report and systematic literature review. J. Pediatric Infect. Dis. Soc. 9, 100-105. doi: 10.1093/jpids/piz039

Clausen, P., Aarestrup, F. M., and Lund, O. (2018). Rapid and precise alignment of raw reads against redundant databases with KMA. BMC Bioinformatics 19:307. doi: 10.1186/s12859-018-2336-6

Delcher, A. L., Phillippy, A., Carlton, J., and Salzberg, S. L. (2002). Fast algorithms for large-scale genome alignment and comparison. Nucleic Acids Res. 30, 2478-2483. doi: 10.1093/nar/30.11.2478

Dou, H., Wang, H., Xie, S., Chen, X., Xu, Z., and Xu, Y. (2017). Molecular characterization of Cryptococcus neoformans isolated from the environment in Beijing, China. Med. Mycol. 55, 737-747. doi: 10.1093/mmy/myx026
Saudi Arabia. Clinical microbiology laboratories should be also prepared for other opportunistic Cryptococcus species, which were also isolated from the same environmental specimens. Less common opportunistic yeast species should be considered when identifying clinical isolates from susceptible patients. It is important to update the fungal identification databases with more whole-genome sequence data to improve the identification of less common opportunistic yeast species.

\section{DATA AVAILABILITY STATEMENT}

The datasets presented in this study can be found in online repositories. The names of the repository/repositories and accession number(s) can be found below: https://www.ncbi.nlm. nih.gov/bioproject/, PRJNA725418.

\section{AUTHOR CONTRIBUTIONS}

E-SK and BSir performed all of the laboratory work. AA, HF, E-SK, and BSir contributed to conception and design of the study. MD, BSin, and MA collected all samples. All authors contributed to manuscript revision, read, and approved the submitted version.

\section{FUNDING}

This work was funded by the Deanship of Scientific Research, Umm Al Qura University, grant number 43309016.

Ellabib, M. S., Aboshkiwa, M. A., Husien, W. M., D’Amicis, R., and Cogliati, M. (2016). Isolation, identification and molecular typing of Cryptococcus neoformans from pigeon droppings and other environmental sources in Tripoli, Libya. Mycopathologia 181, 603-608. doi: 10.1007/s11046-016-9 996-4

Engelthaler, D. M., Hicks, N. D., Gillece, J. D., Roe, C. C., Schupp, J. M., Driebe, E. M., et al. (2014). Cryptococcus gattii in North American Pacific Northwest: whole-population genome analysis provides insights into species evolution and dispersal. mBio 5, e01464-14. doi: 10.1128/mBio.01464-14

Florio, A. R., Ferrari, S., De Carolis, E., Torelli, R., Fadda, G., Sanguinetti, M., et al. (2011). Genome-wide expression profiling of the response to shortterm exposure to fluconazole in Cryptococcus neoformans serotype A. BMC Microbiol. 11:97. doi: 10.1186/1471-2180-11-97

Ghaderi, Z., Eidi, S., and Razmyar, J. (2019). High prevalence of Cryptococcus neoformans and isolation of other opportunistic fungi from pigeon (Columba livia) droppings in Northeast Iran. J. Avian Med. Surg. 33, 335-339. doi: 10. 1647/2018-370

Gillece, J. D., Schupp, J. M., Balajee, S. A., Harris, J., Pearson, T., Yan, Y., et al. (2011). Whole genome sequence analysis of Cryptococcus gattii from the Pacific Northwest reveals unexpected diversity. PLoS One 6:e28550. doi: 10.1371/ journal.pone.0028550

Girish Kumar, C. P., Prabu, D., Mitani, H., Mikami, Y., and Menon, T. (2010). Environmental isolation of Cryptococcus neoformans and Cryptococcus gattii from living trees in Guindy National Park, Chennai, South India. Mycoses 53, 262-264. doi: 10.1111/j.1439-0507.2009.01699.x

Hasman, H., Saputra, D., Sicheritz-Ponten, T., Lund, O., Svendsen, C. A., FrimodtMoller, N., et al. (2014). Rapid whole-genome sequencing for detection and 
characterization of microorganisms directly from clinical samples. J. Clin. Microbiol. 52, 139-146. doi: 10.1128/JCM.02452-13

Heitman, J., Casadevall, A., Lodge, J. K., and Perfect, J. R. (1999). The Cryptococcus neoformans genome sequencing project. Mycopathologia 148, 1-7. doi: 10.1023/ a: 1007136602930

Kaas, R. S., Leekitcharoenphon, P., Aarestrup, F. M., and Lund, O. (2014). Solving the problem of comparing whole bacterial genomes across different sequencing platforms. PLoS One 9:e104984. doi: 10.1371/journal.pone.010 4984

Larsen, M. V., Cosentino, S., Lukjancenko, O., Saputra, D., Rasmussen, S., Hasman, H., et al. (2014). Benchmarking of methods for genomic taxonomy. J. Clin. Microbiol. 52, 1529-1539. doi: 10.1128/JCM.02981-13

Li, H., and Durbin, R. (2009). Fast and accurate short read alignment with BurrowsWheeler transform. Bioinformatics 25, 1754-1760. doi: 10.1093/bioinformatics/ btp324

Li, H., Handsaker, B., Wysoker, A., Fennell, T., Ruan, J., Homer, N., et al. (2009). The sequence alignment/map format and SAMtools. Bioinformatics 25, 20782079. doi: 10.1093/bioinformatics/btp352

Lockhart, S. R., Roe, C. C., and Engelthaler, D. M. (2016). Whole-genome analysis of Cryptococcus gattii, Southeastern United States. Emerg. Infect. Dis. 22, 10981101. doi: 10.3201/eid2206.151455

Meyer, W. (2015). Cryptococcus gattii in the age of whole-genome sequencing. mBio 6, e01761-15. doi: 10.1128/mBio.01761-15

Mirpourian, S. S., Sharifi, N., Talazadeh, F., Jafari, R. A., and Ghorbanpoor, M. (2021). Isolation, molecular identification, and phylogenetic evaluation of Cryptococcus neoformans isolated from pigeon lofts, psittaciformes, and passeriformes in Ahvaz, Iran. Comp. Immunol. Microbiol. Infect. Dis. 76:101618. doi: 10.1016/j.cimid.2021.101618

Movahed, E., Munusamy, K., Tan, G. M., Looi, C. Y., Tay, S. T., and Wong, W. F. (2015). Genome-wide transcription study of Cryptococcus neoformans H99 clinical strain versus environmental strains. PLoS One 10:e0137457. doi: 10.1371/journal.pone.0137457

Pakshir, K., Zareshahrabadi, Z., Zomorodian, K., Ansari, S., Nouraei, H., and Gharavi, A. (2019). Molecular identification of non-Cryptococcus yeasts associated with pigeon droppings in Shiraz, Southern Iran. Iran J. Vet. Res. 20, 204-208.

Price, M. N., Dehal, P. S., and Arkin, A. P. (2010). FastTree 2-approximately maximum-likelihood trees for large alignments. PLoS One 5:e9490. doi: 10. 1371/journal.pone.0009490

Qishui, O., Ling, J., Ni, L., Bin, Y., and Wen, L. (2012). Comparison of realtime florescence quantitative PCR measurements of VAD1 mRNA with three conventional methods in diagnosis and follow-up treatment of Cryptococcus neoformans infection. Mycoses 55, 326-332. doi: 10.1111/j.1439-0507.2011. 02100.x

Quinlan, A. R., and Hall, I. M. (2010). BEDTools: a flexible suite of utilities for comparing genomic features. Bioinformatics 26, 841-842. doi: 10.1093/ bioinformatics/btq033
Ragupathi, L., and Reyna, M. (2015). Case report of Cryptococcus albidus peritonitis in a peritoneal dialysis patient and a review of the literature. Perit. Dial. Int. 35, 421-427. doi: 10.3747/pdi.2013.00134

Rodriguez-Leguizamon, G., Coque-Burgos, E., Espitia-Castro, O., and Firacative, C. (2020). Fatal fungaemia due to Cryptococcus albidus in an elderly diabetic woman presenting with pleural effusion. Rev. Inst. Med. Trop. Sao Paulo 62:e34. doi: 10.1590/s1678-9946202062034

Satoh, K., Maeda, M., Umeda, Y., Miyajima, Y., and Makimura, K. (2011). Detection and identification of probable endemic fungal pathogen, Cryptococcus gattii, and worldwide pathogen, Cryptococcus neoformans, by real-time PCR. Microbiol. Immunol. 55, 454-457. doi: 10.1111/j.1348-0421.2011.00324.x

Soltani, M., Bayat, M., Hashemi, S. J., Zia, M., and Pestechian, N. (2013). Isolation of Cryptococcus neoformans and other opportunistic fungi from pigeon droppings. J. Res. Med. Sci. 18, 56-60.

Sugita, T., Takashima, M., Ikeda, R., Nakase, T., and Shinoda, T. (2000). Phylogenetic and taxonomic heterogeneity of Cryptococcus humicolus by analysis of the sequences of the internal transcribed spacer regions and $18 \mathrm{~S}$ rDNA, and the phylogenetic relationships of C. humicolus, C. curvatus, and the genus Trichosporon. Microbiol. Immunol. 44, 455-461.

Vajpeyi, S., and Chandran, K. (2016). Draft genome sequence of the oleaginous yeast Cryptococcus albidus var. albidus. Genome Announc. 4, e00390-16. doi: 10.1128/genomeA.00390-16

Wu, Y., Du, P. C., Li, W. G., and Lu, J. X. (2012). Identification and molecular analysis of pathogenic yeasts in droppings of domestic pigeons in Beijing, China. Mycopathologia 174, 203-214. doi: 10.1007/s11046-012-9536-9

Yong, X., Yan, Z., Xu, L., Zhou, J., Wu, X., Wu, Y., et al. (2016). Genome sequence of a microbial lipid producing fungus Cryptococcus albidus NT2002. J. Biotechnol. 223, 6-7. doi: 10.1016/j.jbiotec.2016.02.026

Conflict of Interest: The authors declare that the research was conducted in the absence of any commercial or financial relationships that could be construed as a potential conflict of interest.

Publisher's Note: All claims expressed in this article are solely those of the authors and do not necessarily represent those of their affiliated organizations, or those of the publisher, the editors and the reviewers. Any product that may be evaluated in this article, or claim that may be made by its manufacturer, is not guaranteed or endorsed by the publisher.

Copyright (c) 2021 Sirag, Khidir, Dumyati, Sindi, Alsinnari, Faidah and Ahmed. This is an open-access article distributed under the terms of the Creative Commons Attribution License (CC BY). The use, distribution or reproduction in other forums is permitted, provided the original author(s) and the copyright owner(s) are credited and that the original publication in this journal is cited, in accordance with accepted academic practice. No use, distribution or reproduction is permitted which does not comply with these terms. 